\title{
The Membrane Potential Waveform of Bursting Pacemaker Neurons Is a Predictor of Their Preferred Frequency and the Network Cycle Frequency
}

\author{
Hua-an Tseng ${ }^{1}$ and Farzan Nadim ${ }^{1,2}$ \\ ${ }^{1}$ Federated Department of Biological Sciences, Rutgers University, and 2Department of Mathematical Sciences, New Jersey Institute of Technology, Newark, \\ New Jersey 07102
}

\begin{abstract}
Many oscillatory networks involve neurons that exhibit intrinsic rhythmicity but possess a large variety of voltage-gated currents that interact in a complex fashion, making it difficult to determine which factors control frequency. Yet these neurons often have preferred (resonance) frequencies that can be close to the network frequency. Because the preferred frequency results from the dynamics of ionic currents, it can be assumed to depend on parameters that determine the neuron's oscillatory waveform shape. The pyloric network frequency in the crab Cancer borealis is correlated with the preferred frequency of its bursting pacemaker neurons anterior burster and pyloric dilator (PD). We measured the preferred frequency of the PD neuron in voltage clamp, which allows control of the oscillation voltage range and waveforms (sine waves and realistic oscillation waveforms), and showed that (1) the preferred frequency depends on the voltage range of the oscillating voltage waveform; (2) the slope of the waveform near its peak has a strongly negative correlation with the preferred frequency; and (3) correlations between parameters of the PD neuron oscillation waveform and its preferred frequency can be used to predict shifts in the network frequency. As predicted by these results, dynamic clamp shifts of the upper or lower voltage limits of the PD neuron waveform during ongoing oscillations changed the network frequency, consistent with the predictions from the preferred frequency. These results show that the voltage waveform of oscillatory neurons can be predictive of their preferred frequency and thus the network oscillation frequency.
\end{abstract}

\section{Introduction}

Oscillatory neuronal networks usually produce their output in a certain frequency range. The network frequency can be determined by the properties of individual neurons and the interaction between neurons (Marder and Calabrese, 1996) and, in some systems, solely by the oscillation frequency of the pacemaker neurons (Marder and Bucher, 2001). Previous studies have shown that the frequency of a bursting network oscillation can be strongly correlated with the preferred (resonance) frequency of the neurons within the network (Tohidi and Nadim, 2009). An important characteristic of a bursting neuron is its slow oscillation membrane potential, which can be represented as a unitary waveform. We explored the question of how the parameters of this waveform affect the preferred frequency of the neuron and, potentially, the frequency of the network activity.

The crustacean pyloric network generates a tri-phase rhythmic activity around $1 \mathrm{~Hz}$. Many pyloric neurons, including the pacemaker neurons anterior burster $(\mathrm{AB})$ and pyloric dilator (PD), show a frequency-dependent response to oscillatory in-

Received April 8, 2010; revised June 26, 2010; accepted June 30, 2010

This work was supported by National Institutes of Health Grant MH-60605. We gratefully acknowledge Jorge Golowasch and Isabel Soffer for their critical comments on the manuscript.

Correspondence should be addressed to Farzan Nadim, Department of Mathematical Sciences, New Jersey Institute of Technology, 323 Martin Luther King Boulevard, Newark, NJ 07102. E-mail: farzan@njit.edu.

DOI:10.1523/JNEUROSCI.1818-10.2010

Copyright $\odot 2010$ the authors $\quad 0270-6474 / 10 / 3010809-11 \$ 15.00 / 0$ puts, resulting in a preferred frequency at which the neuron shows the highest impedance (Tohidi and Nadim, 2009). Here we examine the hypothesis that changes in the PD neuron waveform shape can shift its preferred frequency and, consequently, the frequency of the pyloric network activity as a whole. Thus, by knowing how defining parameters of the PD neuron waveform influence the preferred frequency, we should be able to predict shifts in the pyloric network frequency as a result of physiological inputs or experimental manipulations that reshape the waveform.

The oscillation waveform of the PD neuron is defined by multiple parameters, including the voltage minimum and maximum, duty cycle, peak phase, and the rise and fall slopes. In an ongoing pyloric activity, these parameters are defined by a variety of factors. For instance, synaptic inhibition from the follower lateral pyloric (LP) neuron alters the lower bound of the PD waveform (Thirumalai et al., 2006), whereas voltagegated ionic currents, such as the A current, contribute to the process of rebound by shaping the rising slope of the waveform and its duty cycle (Tierney and Harris-Warrick, 1992; MacLean et al., 2003). Additionally, these parameters are subjected to neuromodulation (Kloppenburg et al., 1999; Peck et al., 2001; Thirumalai et al., 2006).

We demonstrate that changes in the preferred frequency of a neuron can be predicted by examining parameters that determine its voltage waveform. By measuring the preferred frequency with the voltage-clamp technique, we find that the voltage range 
of the oscillation and a few descriptive parameters of the PD neuron waveform, including its rising slope near peak, are strongly correlated with its preferred frequency. Using the dynamic-clamp technique, we introduced artificial ionic currents in the PD neuron during its ongoing oscillation and found that changing the waveform parameters shift the network frequency in a manner consistent with the predicted shifts in the preferred frequency. As such, novel changes in the network frequency can be predicted by examining the effect of ionic or synaptic currents on the pacemaker neuron's waveform shape. These results demonstrate the ability to predict shifts in the network frequency in response to changes in certain parameters characterizing the pacemaker neuron waveform, regardless of how these parameters are altered.

\section{Materials and Methods}

Experimental protocols. Adult male crabs (Cancer borealis) were purchased from local distributors and kept in aquaria filled with chilled artificial sea water until use. Before dissection, the crabs were anesthetized by putting them on ice for at least $20 \mathrm{~min}$. The dissection was done using standard protocols as described in previous studies (Tohidi and Nadim, 2009). After dissection, the nervous system, including the commissural ganglia, the esophageal ganglion, the stomatogastric ganglion (STG), the nerves connecting these ganglia, and motor nerves, were pinned down in a $100 \mathrm{~mm}$ Petri dish coated with the silicon elastomer Sylgard (Dow Corning). The STG was then desheathed to expose the neurons for impalement. During the experiment, the whole dish was superfused with normal crab saline $(11 \mathrm{~mm} \mathrm{KCl}, 440 \mathrm{~mm} \mathrm{NaCl}, 13 \mathrm{~mm}$ $\mathrm{CaCl}_{2} \cdot 2 \mathrm{H}_{2} \mathrm{O}, 26 \mathrm{~mm} \mathrm{MgCl}_{2} \cdot 6 \mathrm{H}_{2} \mathrm{O}, 11.2 \mathrm{~mm}$ Trizma base, $5.1 \mathrm{~mm}$ maleic acid; $\mathrm{pH}$ 7.4) maintained at $10-13^{\circ} \mathrm{C}$. The $\mathrm{PD}$ neurons were identified by matching their intracellular activity with the extracellular action potentials on the corresponding motor nerves.

The electrodes were prepared by using the Flaming-Brown micropipette puller (P97; Sutter Instrument) and filled with $0.6 \mathrm{M} \mathrm{K}_{2} \mathrm{SO}_{4}$ and 0.02 $\mathrm{M} \mathrm{KCl}$. For current injection, the resistance of the electrode was kept at $10-20 \mathrm{M} \Omega$; for membrane potential measurements, the resistance of the electrode was $20-35 \mathrm{M} \Omega$. Extracellular recording from the motor nerves was done using a differential AC amplifier model 1700 (A-M Systems) and intracellular recording was done with Axoclamp 2B amplifiers (Molecular Devices).

Measurements of the preferred frequency. The two PD neurons are electrically coupled to the $\mathrm{AB}$ neuron with which they form the pyloric pacemaker kernel. We have previously shown that the measurement of preferred frequency in either of these neuron types is not influenced by the contribution from their electrically coupled partners (Tohidi and Nadim, 2009). However, it should be noted that in all manipulations in the current study, the $\mathrm{AB}$ neuron is present and may contribute to some of the observed results. In particular, the $\mathrm{AB}$ neuron is essential for the generation of the pyloric network rhythm and therefore the effects of current injections into the PD neuron on the pyloric frequency during the ongoing rhythm most probably involve indirect influences on the $\mathrm{AB}$ neuron.

To measure the preferred frequency, after identifying the PD neuron, we used $10^{-7} \mathrm{M}$ tetrodotoxin (TTX; Biotium) to remove the neuromodulatory inputs and thus the endogenous network activity (Raper, 1979; Luther et al., 2003). The preferred frequency was measured in voltage clamp or current clamp using the software Scope (version 7.75). All experiments were done with two electrodes, one used for injecting current and the other for recording the membrane potential. In the current-clamp experiments, the injected current used was a sweeping-frequency sinusoidal (impedance amplitude profile or ZAP) function. A similar ZAP function was used in the voltage-clamp experiments to clamp the membrane potential. The ZAP function was calculated as follows:

$$
\operatorname{ZAP}(t)=A \sin (2 \pi f(t) t)
$$

where $A$ is the amplitude of the oscillation and $f(t)$ is a monotonically increasing function that determines the range of the sweeping frequencies (in $\mathrm{Hz}$ ) and is defined as

$$
\begin{gathered}
f(t)=10^{-3} \frac{F_{\min }}{L t}(\exp (L t)-1) \\
L=\ln \left(\frac{F_{\max }}{F_{\min }}\right) / \mathrm{dur},
\end{gathered}
$$

where $F_{\max }$ and $F_{\min }$ are the maximum and minimum swept frequencies, respectively; dur is the duration of the sweep; and $L$ is the rate of the exponential rise in frequency (the exponential rise in frequency is used so that lower frequencies are sampled sufficiently). Because time was measured in milliseconds, the scaling factor $10^{-3}$ was used to convert the frequency units to $\mathrm{Hz}$. All experiments were done with $F_{\min }=0.1 \mathrm{~Hz}$, $F_{\max }=4 \mathrm{~Hz}$, and dur $=100 \mathrm{~s}$. To avoid transients at the beginning of the oscillations, the ZAP waveform was preceded with three cycles of a sinusoidal waveform injected at the lowest frequency $F_{\min }$, which transitioned smoothly into the ZAP function, bringing the total duration of each sweep to $130 \mathrm{~s}$. The first sinusoidal waveform was phase shifted to start at its minimum value. Henceforth, we use the term ZAP function to refer to the total injected function, including the first three sinusoidal waveforms.

In the current-clamp experiments, the amplitude of the ZAP function current was adjusted to produce a $30 \mathrm{mV}$ difference in membrane potential when the PD neuron was oscillating at $0.1 \mathrm{~Hz}$. To make the results comparable to those from the voltage-clamp recordings, when necessary, we also injected a bias DC current to bring the baseline membrane potential to $-60 \mathrm{mV}$.

The voltage-clamp experiments were done in two-electrode voltageclamp mode. In each sweep, the membrane potential was clamped first at a holding value (equal to the minimum value of the ZAP function), followed by the ZAP function. The voltage range of oscillation varied depending on the experiment. The PD neuron produces bursting oscillations at a frequency of $\sim 1 \mathrm{~Hz}$ with a slow-wave profile that ranges approximately between -60 and $-30 \mathrm{mV}$. We therefore focused our study around these voltage and frequency ranges (Tohidi and Nadim, 2009). In the experiments in which the effect of the lower bound was examined, the lower bound was shifted by $\pm 10 \mathrm{mV}(-70,-60$, and -50 $\mathrm{mV}$ ) while the upper bound was kept at $-30 \mathrm{mV}$. When examining the effect of the upper bound, this bound was shifted by $\pm 4 \mathrm{mV}(-34,-30$, and $-26 \mathrm{mV}$ ) while the lower bound was kept at $-60 \mathrm{mV}$. In a subset of experiments, we also did sweeps in which the upper bound was shifted by $\pm 10 \mathrm{mV}$ and the frequency range was between 0.1 and $10 \mathrm{~Hz}$. However, setting the upper bound too high $(-20 \mathrm{mV})$ resulted in a second impedance peak in the higher frequency range $(>5 \mathrm{~Hz})$, whereas setting the upper bound too low $(-40 \mathrm{mV})$ often resulted in a shift of the peak to zero frequency (Tohidi and Nadim, 2009). We therefore limited the shift of the upper bound to $\pm 4 \mathrm{mV}$, which resulted, on average, in a shift of $f_{\max }$ that was similar to that seen when the lower bound was shifted by $\pm 10 \mathrm{mV}$.

The impedance profile was generated with a Matlab (MathWorks) script. For each cycle of the recording, the script measured the frequency and the amplitudes of the changes in voltage and current, and the impedance $[Z(f)]$ was calculated as a ratio of the voltage and current amplitudes as a function of frequency. Alternatively, $Z(f)$ can be measured as the ratio of the Fourier transforms of the voltage and current traces (Hutcheon and Yarom, 2000). The preferred frequency is defined as the frequency at which the impedance power $|Z(f)|$ is maximal. In Results, below, we refer to the impedance power as impedance.

Dynamic clamp. The Dynamic Clamp software (version 1.55) was used to produce artificial ionic conductance to shift the voltage range of an oscillating PD neuron during its ongoing network activity. The current was injected into the PD neuron in discontinuous current-clamp mode. In the upper-bound shift experiments, the artificial current had a reversal potential at $0 \mathrm{mV}$ and activated when the PD membrane potential was above a given threshold; in the lower-bound shift experiments, the artificial current had a reversal potential at $-80 \mathrm{mV}$ and activated when the $\mathrm{PD}$ membrane potential was below a given threshold. In each experiment, we used two different thresholds. The lower threshold was set at 
the voltage at which the inhibitory synapse from the LP neuron activated $(\sim-55 \mathrm{mV})$, whereas the higher threshold was always set $10 \mathrm{mV}$ higher than the lower threshold. The dynamic clamp current was assumed to activate with first-order activation kinetics and no inactivation:

$$
I_{\text {dynamic }}=g_{\max } m\left(v-E_{\text {rev }}\right) .
$$

The activation variable followed the equations

$$
\begin{gathered}
\frac{d m}{d t}=\left(m_{\infty}(v)-m\right) / \tau_{\mathrm{m}}(v) \\
m_{\infty}(v)=1 /\left(1+\exp \frac{v-v_{\mathrm{th}}}{k}\right) .
\end{gathered}
$$

Here, $v_{\text {th }}$ is the activation threshold, $k$ was set to $-1 \mathrm{mV}$ to produce a sharp activation curve, and the time constant $\left(\tau_{\mathrm{m}}\right)$ was set to a constant value of $1 \mathrm{~ms}$. By adjusting the maximal conductance $g_{\max }$ (range, 5-35 $\mathrm{nS}$ ), we could move the upper bound or the lower bound of the PD neuron membrane potential to different levels. In the upper and lower bound shift experiments, we limited the value of $g_{\max }$ so that only the upper or lower bounds, respectively, were affected. This restriction resulted in a smaller possible range of $g_{\max }$ values for the low threshold activation inward current and thus a more limited shift in the upper bound when compared with the high threshold activation current.

Generation of the representative realistic PD neuron waveforms. The representative unitary waveforms were extracted from the experimental recordings using the Readscope software (version 7.75) as described below. Five consecutive cycles of each selected PD neuron recording were analyzed. The voltage trace was low-pass filtered at $10 \mathrm{~Hz}$ to reveal the slow-wave oscillation. The average membrane potential in the five cycles was used as the voltage threshold to align and average these cycles and produce a single averaged waveform. The averaged waveform was sampled at 1000 points and rescaled to be between 0 and 1 . Finally, the waveform was shifted so that the minimum point 0 became the start point of the waveform. These representative unitary waveforms were then used in the waveform analysis and in the experiments. The experiments in which the waveform was used to voltage clamp the PD neuron and measure the corresponding preferred frequency were performed by replacing the sine function in Equation 1 with the unitary waveform using the Scope software.

Waveform analysis. The waveform analysis was done using Matlab scripts. The waveform duty cycle was defined as the fraction of time that the waveform was above its mean value. We also calculated the portions of waveform $>75 \%$ or $<25 \%$ of the peak value. The peak phase is the phase at which the maximum value (1) occurs when the minimum (0) is used as the reference point. Area refers to the area underneath the waveform curve where the duration of the waveform is normalized to 1 . The various slopes were calculated from different parts of the waveform after dividing the waveform into a rising and a falling interval. The rising interval is the portion of the waveform from between the start point 0 and the peak point 1 and the falling interval is between the peak and the end point. Each interval was divided into different slope ranges. The rising slopes used were as follows: $0-100,0-25,25-50,50-75$, and $75-100 \%$; the falling slopes were as follows: $100-0,100-75,75-50,50-25$, and $25-0 \%$. These parameters were not meant to be independent or exhaustive descriptions of the waveforms. These were selected from among a larger set of parameters because they provide a relatively distinct and comprehensive description of the waveforms.

The software tools Scope, Readscope, and Dynamic Clamp were developed in the Nadim laboratory and are available for download at http://stg.rutgers.edu/software.

Statistical analysis. All statistical analysis was performed in SigmaStat (version 2.03, Aspire Software International). Significance was evaluated by comparing with the $\alpha$ value of 0.05 .

\section{Results}

A previous study from our lab showed that the pacemaker PD neuron bursting frequency, and therefore the pyloric network frequency $\left(f_{\text {pyloric }}\right)$, is strongly correlated with the preferred fre-
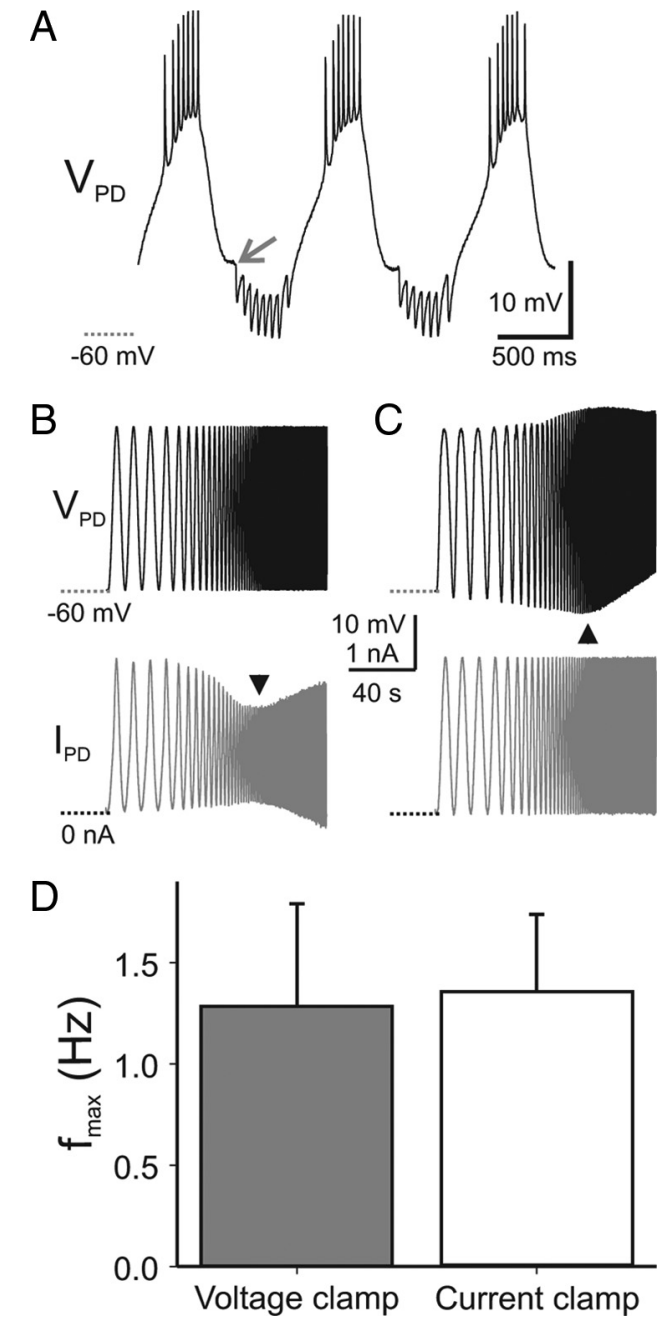

Figure 1. The preferred frequency of the PD neuron measured under voltage-clamp or current-clamp conditions. A, During the pyloric rhythm, the ongoing oscillation of the PD neuron $\left(V_{\mathrm{PD}}\right)$ has a slow waveform ranging $\sim-60$ to $-30 \mathrm{mV}$. A prominent characteristic of the PD waveform is the kink produced by the onset of the IPSPs from the follower $L P$ neuron (arrow). $B$, The membrane potential and the injected current $\left(I_{\mathrm{PD}}\right)$ were recorded when the PD neuron was voltage clamped with a ZAP function ranging between -60 and $-30 \mathrm{mV}$ and sweeping frequencies of $0.1-4 \mathrm{~Hz}$. Arrowhead, Preferred frequency where the impedance is maximal. $\boldsymbol{C}$, The preferred frequency was measured in current-clamp condition by injecting a ZAP current with sweeping frequencies of $0.1-4$ $\mathrm{Hz}$. The current amplitude was adjusted so that at the lowest frequency $(0.1 \mathrm{~Hz})$, the resulting membrane potential oscillations ranged from -60 to $-30 \mathrm{mV}$. Arrowhead, Preferred frequency. $\boldsymbol{D}$, The preferred frequencies showed no difference when measured under the voltage-clamp or the current-clamp conditions $(N=7$, paired Student's $t$ test, $p=0.742$ ). Error bars show SD values.

quency $\left(f_{\max }\right)$ of the PD neurons (Tohidi and Nadim, 2009). We now explore the parameters of the slow-oscillation waveform underlying the bursting activity of PD neurons that affect $f_{\max }$ and examined whether these parameters also change $f_{\text {pyloric }}$. Our results are divided into four sets of experiments. First, we show that the preferred frequency measured in voltage clamp is statistically identical to the frequency traditionally measured in current clamp. Voltage-clamp measurements allow for the precise determination of the dependence of $f_{\max }$ on the voltage range and oscillation waveform shape. Second, we demonstrate that shifts in the upper or lower limits of the PD neuron voltage range result in a shift in $f_{\max }$ in the same direction. Third, using the dynamicclamp technique, we add inward and outward voltage-dependent 
currents to shift the upper and lower limits, respectively, of the PD neuron voltage range during its ongoing pyloric oscillation and show that these manipulations typically result in a shift in the $f_{\text {pyloric }}$, similar to that of $f_{\max }$. However, the dependence of $f_{\text {pyloric }}$ on the upper bound of the voltage waveform is sensitive to the activation threshold for the dynamicclamp current. Finally, we measure $f_{\max }$ using different prerecorded realistic PD neuron waveforms and show that there are correlations between $f_{\max }$ and certain parameters that determine the waveform shape.

\section{Measurement of impedance in voltage clamp}

The PD neuron exhibits a preferred frequency when its impedance is measured by injecting a ZAP current of fixed amplitude that allows the membrane potential to oscillate between the limits of $\sim-60$ and $\sim-30 \mathrm{mV}$ (Tohidi and Nadim, 2009). These values provide an approximation of the slow-wave oscillation of the PD neuron bursting waveform during ongoing pyloric activity (Fig. 1A). Using ZAP current injection is the traditional way of measuring neuronal impedance and demonstrating the presence of resonance (Puil et al., 1986; Hutcheon and Yarom, 2000). However, in the current study, we were interested in how the properties of the PD neuron waveform, such as its voltage range and shape, affect $f_{\text {max }}$. To change the voltage range in a controlled manner, we measured the impedance of the PD neuron in voltage clamp (see Materials and Methods). After the TTX treatment, the PD neuron was voltageclamped with a ZAP function waveform sweeping frequencies between 0.1 and 4 $\mathrm{Hz}$ and the voltage range between -60 and $-30 \mathrm{mV}$, and the injected current was recorded (Fig. 1B). The impedance peak in such a measurement corresponds to the frequency at which the injected current is minimal. One important question was whether the impedance measured in voltage clamp would produce the same value of $f_{\text {max }}$ as that measured in current clamp. To address this question, we did paired current- and voltage-clamp impedance measurements of the PD neuron. Under current-clamp conditions, the membrane potential of the PD neuron at the lowest frequency of $0.1 \mathrm{~Hz}$ was matched to the voltage-clamp range of $-60--30 \mathrm{mV}$ (Fig. 1C). These comparisons showed that the values of $f_{\max }$ obtained under the current-clamp or voltage-clamp conditions are statistically identical (Student's $t$ test, $N=7, p=0.742$ ) (Fig. $1 D$ ). In all subsequent experiments described in our study, we measured $f_{\max }$ in voltage clamp.
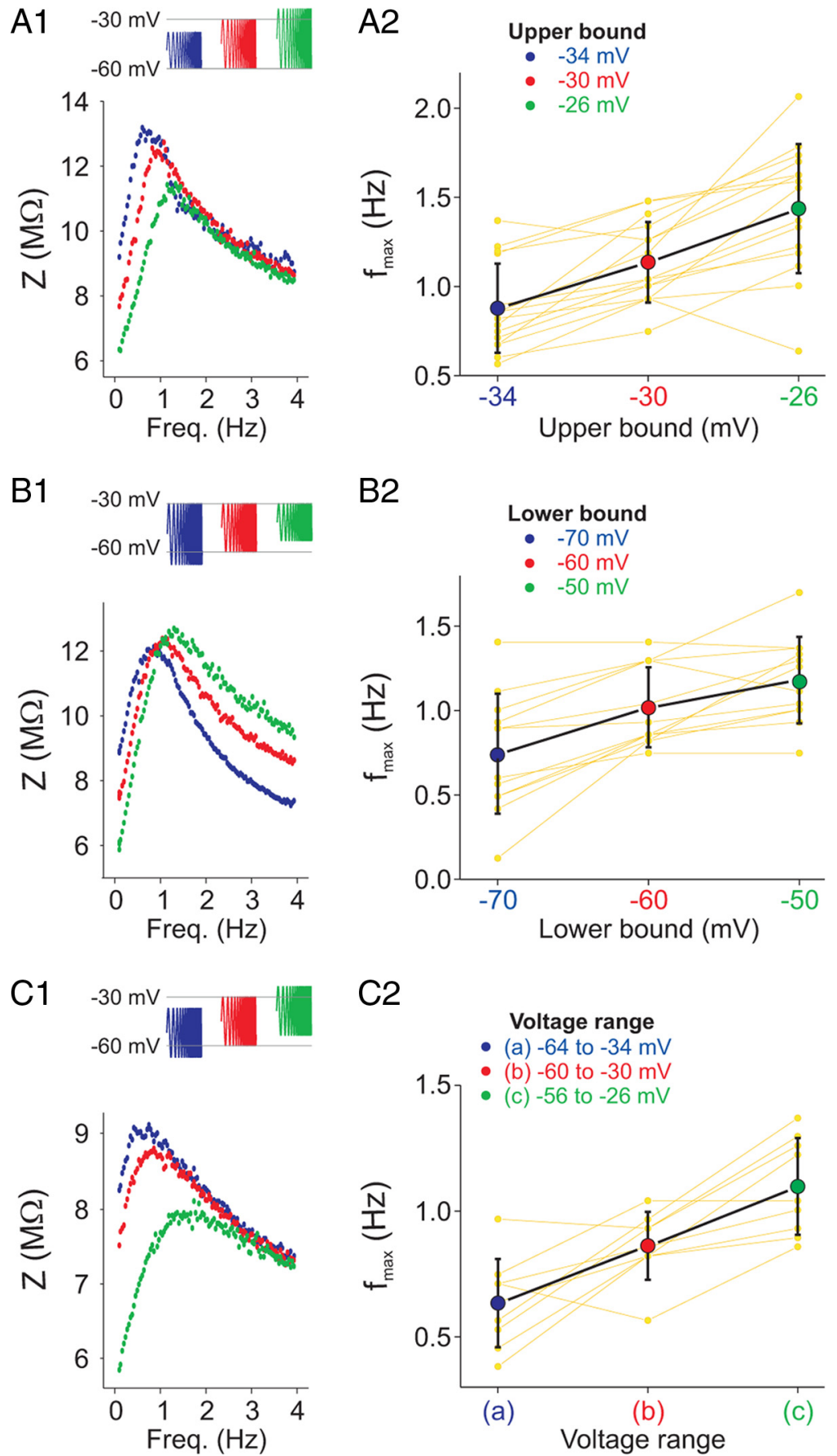

Figure 2. When the voltage range of oscillation was increased or decreased, the preferred frequency (Freq.) shifted in the same direction. $\boldsymbol{A 1}$, The impedance profiles of the PD neuron measured in voltage clamp using ZAP functions with a fixed lower bound $(-60 \mathrm{mV})$ and various upper bounds (inset, blue, $-34 \mathrm{mV} ;$ red, $-30 \mathrm{mV}$; green, $-26 \mathrm{mV})$. A2, The preferred frequency increases when the upper bound is increased $(N=15 ; p<0.001)$. B1, The impedance profiles with a fixed upper bound $(-30 \mathrm{mV})$ and various lower bounds (inset, blue, $-70 \mathrm{mV}$; red, $-60 \mathrm{mV}$; green, $-50 \mathrm{mV}$ ). $\boldsymbol{B 2}$, The preferred frequency also increases when the lower bound is increased $(N=12 ; p<0.05)$. $C 1$, The impedance profiles with a shifting voltage range, as indicated in the inset and legend. C2, The preferred frequency increased with the voltage range of oscillation $(N=9 ; p<0.001)$. Yellow data points in $\boldsymbol{A 2}$, $\mathbf{B 2}$, and $\mathbf{C}$ show values in individual experiments. Error bars show SD values.

\section{The effect of the PD neuron voltage range on $f_{\max }$}

During the ongoing pyloric network activity, many factors may affect the upper and lower bounds of the bursting oscillations the PD neuron. These include the inhibitory synaptic input from the follower LP neuron (Thirumalai et al., 2006) (Fig. 1A) as well as the modulatory environment (Marder and Eisen, 1984; Kloppenburg et al., 1999; Thirumalai and Marder, 2002; Goaillard et al., 2004). 
A1
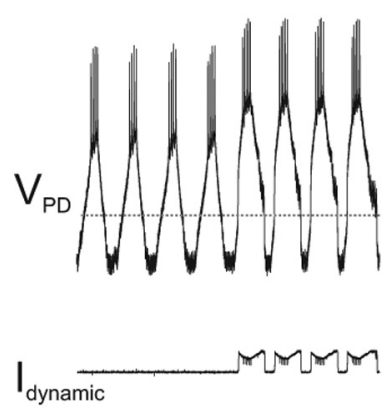

$10 \mathrm{mV}$

B1

$10 \mathrm{nA}$

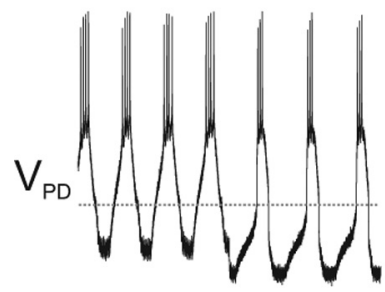

Idynamic $v$ inir
A2

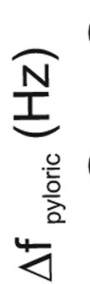

0.00

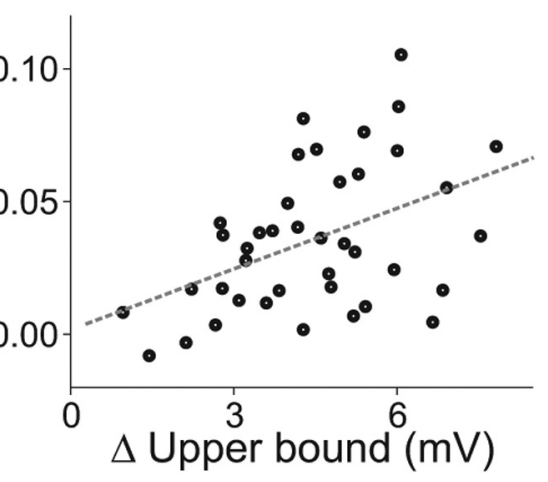

0

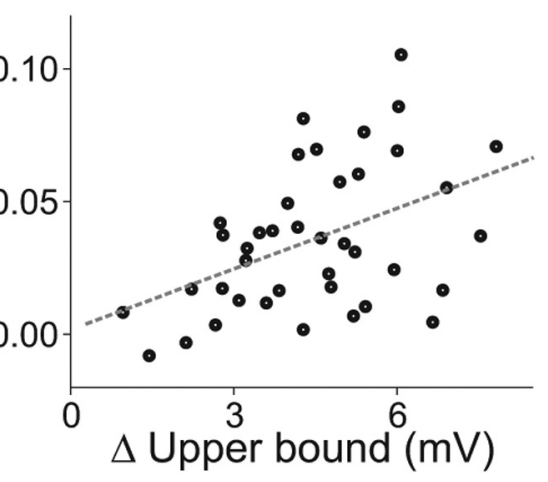

B2

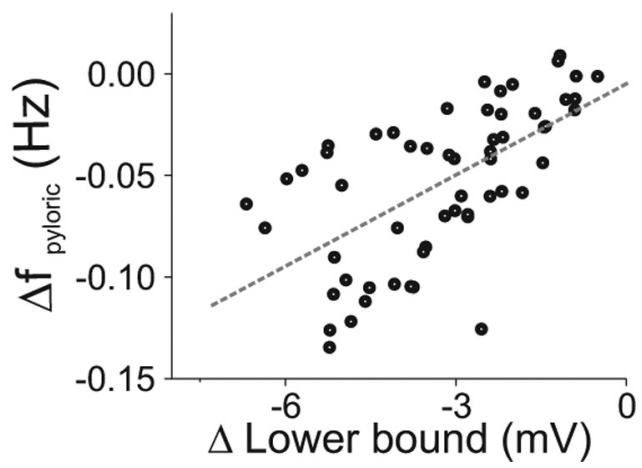

Figure 3. Altering the voltage range of the PD neuron $\left(V_{\mathrm{PD}}\right)$ oscillation shifts the pyloric network frequency $f_{\text {pyloric }} \cdot \boldsymbol{A}$, The upper bound of the PD membrane potential in an ongoing pyloric network was modified by adding a dynamic-clamp inward current $\left(I_{\text {dynamic }}\right) . \mathbf{A 1}$, The upper bound of the voltage range shifted to higher values during the dynamic-clamp current injection. $\mathbf{A 2}, f_{\text {pyloric }}$ increased as the upper bound of the voltage oscillations increased. Dashed line shows the linear fit $(r=0.45, p<0.05)$. B, The lower bound of the PD membrane potential was modified by adding a dynamic-clamp outward current. $\boldsymbol{B} 1$, The lower bound of the voltage range shifted to lower values with the dynamic clamp current injection. $\boldsymbol{B} \mathbf{2}, f_{\text {pyloric }}$ decreased as the lower bound of the voltage oscillations decreased. Dashed line shows the linear fit $(r=0.61, p<0.01)$. The lines in $\mathbf{A} 1$ and $\mathbf{B} 1$ indicate the threshold voltage for the activation of the dynamic-clamp current. Data points in $\mathbf{A} \mathbf{2}$ and $\boldsymbol{B} \mathbf{2}$ are multiple runs in six experiments performed with different $g_{\max }$ values in dynamic clamp.

Thus, we decided to examine possible effects of the waveform voltage range on the $f_{\max }$ value in the $\mathrm{PD}$ neuron. The waveform voltage range can be altered by shifting the upper bound, the lower bound, or both. We first explored the change in the upper bound while keeping the lower bound at a fixed voltage of -60 $\mathrm{mV}$. As our control value, we used an upper bound voltage of $-30 \mathrm{mV}$ and then increased or decreased this upper bound by 4 $\mathrm{mV}$ (Fig. $2 A 1$, top). In a preliminary set of experiments, we also examined the effect of changing the upper bound by $10 \mathrm{mV}$ and a wider frequency range $(0.1-10 \mathrm{~Hz})$, but decided to use the smaller range for reasons explained in the Materials and Methods section.

An example of the impedance profiles for these three different upper bounds is shown in Figure $2 A 1$. When the upper bound was shifted to a higher value of $-26 \mathrm{mV}$, the average $f_{\max }$ increased by $\sim 27 \%$ compared with the control value. Similarly, when the upper bound was shifted to a lower value of $-34 \mathrm{mV}$, the average $f_{\max }$ decreased by $\sim 23 \%$ compared with the control. Figure 2 A2 summarizes this effect for 15 experiments. showing that changing the upper bound of the voltage range significantly shifts $f_{\max }$ in the same direction $(N=15$; one-way ANOVA; $p<0.001)$.

Adjusting only the lower bound of the oscillation had a similar effect on $f_{\text {max }}$. An example of the impedance profiles for three different lower bounds is shown in Figure $2 B 1$. When the lower bound was increased from $-60 \mathrm{mV}$ to $-50 \mathrm{mV}, f_{\max }$ increased by

$\sim 18 \%$ compared with the control value. Likewise, a decrease of the lower bound to $-70 \mathrm{mV}$ resulted in a shift of $f_{\max }$ by $\sim 30 \%$ compared with control $(N=12$; one-way ANOVA; $p<0.05$ ) (Fig. 2 B2).

To examine which is the more effective way of shifting the preferred frequency, we compared the sensitivity of $f_{\max }$ to variations of the upper and lower bounds of the voltage range. We did this by comparing the total change in $f_{\max }$ value divided by the voltage change during the upper or lower bound shifts. We found that changing the upper bound of the voltage range was significantly more effective in changing the $f_{\max }$ value (upper bound $\Delta f_{\max }$ l $\Delta V=0.07 \pm 0.044 \mathrm{~Hz} / \mathrm{mV}$; lower bound $\Delta f_{\max } / \Delta V=0.02 \pm 0.017 \mathrm{~Hz} / \mathrm{mV} ; p=$ 0.001 , Student's $t$ test).

Finally, we shifted both upper and lower bounds by $4 \mathrm{mV}$ in the same direction. An example of the frequency-dependent profiles for these different voltage windows is shown in Figure 2C1 (top). Note that with this manipulation, the amplitude of the membrane potential oscillations remains $30 \mathrm{mV}$ in all three cases. Shifting the voltage range up by $4 \mathrm{mV}, f_{\text {max }}$ increased by $\sim 29 \%$ and a downward shift of $4 \mathrm{mV}$ resulted in an $\sim 25 \%$ decrease of $f_{\max }(N=9$; one-way ANOVA; $p<0.001)$ (Fig. 2C2). The change in $f_{\max }$ due to the upward shift of the voltage range was not significantly different from the change from increasing the upper bound alone (Student's $t$ test, $p=0.876$; increase in the upper bound by $4 \mathrm{mV}, \Delta f_{\max }=26.9 \pm$ $25.5 \%, N=15$; shifting up the voltage range by $4 \mathrm{mV}, \Delta f_{\max }=28.5 \pm 18.9 \%, N=9$ ). It is possible that the lack of difference is due to the low sensitivity of $f_{\max }$ to the lower bound of the oscillation, such that moving the lower bound up by $4 \mathrm{mV}$ in the shift experiment does not produce a significant additional effect. In general, the $f_{\max }$ in the PD neuron shifted in the same direction with the voltage range of oscillation; the higher the voltage range, the higher the $f_{\max }$.

\section{The effect of the PD neuron voltage range on the pyloric frequency}

The preferred frequency $f_{\max }$ of the $\mathrm{PD}$ neuron has been shown to be correlated with the pyloric network frequency (Tohidi and Nadim, 2009). Our data showed that when the PD neuron was driven with an oscillatory stimulus, the measured $f_{\max }$ was influenced by the upper and lower bounds of the membrane potential oscillations. We therefore posed the following hypothesis: Factors that shift the preferred frequency $\left(f_{\max }\right)$ in one direction also change the ongoing pyloric frequency $\left(f_{\text {pyloric }}\right)$ in the same direction. To examine this hypothesis, we first explored whether modifications in the voltage range of the oscillating PD neuron during the ongoing rhythm had an effect on $f_{\text {pyloric }}$. Our prediction was that shifting upward the upper or lower bound of the PD neuron voltage range during the ongoing pyloric oscillation would lead to an increase in $f_{\text {pyloric }}$, consistent with the effects of the voltage range on $f_{\max }$. 
To change the upper bound of the voltage in an oscillating PD neuron during an ongoing pyloric activity, we used the dynamicclamp technique to generate an artificial voltage-gated inward current $\left(I_{\text {dynamic }}\right)$ that activated when the membrane potential of the PD neuron was above a predetermined threshold. Because, in different preparations, PD neurons have slightly different voltage ranges in their ongoing oscillation, we needed to decide on a voltage threshold that was tied to some feature of the PD neuron waveform. One prominent feature of the PD neuron oscillation waveform is the IPSPs it receives from the follower LP neuron (Fig. 1A). Thus, we used the voltage at the onset of these IPSPs (referred to as the low threshold, $V_{\text {low }}$ ) (Fig. $1 A$, arrow) as a reference voltage and the activation threshold for $I_{\text {dynamic }}$ was set to be at this value. Because of the sharp activation threshold of $I_{\text {dynamic }}$, only the upper bound of the PD neuron voltage range was increased whereas the lower bound remained unchanged (Fig. 3A1). By adjusting the maximal conductance value of $I_{\text {dynamic, we }}$ were able to push the upper bound of the PD oscillation to different voltage levels. In each run, we injected the artificial voltagegated current into the PD neuron during the ongoing oscillation for $20 \mathrm{~s}$ and compared the resulting $f_{\text {pyloric }}$ and voltage ranges before and during injection. The traces in which the lower bound of the voltage range was also shifted and were discarded. As predicted, the inclrease in the upper bound of the PD oscillation resulted in higher pyloric network frequencies $(N=6 ; r=0.45$; $p<0.05$ ) (Fig. 3A2).

Similarly, in separate sets of runs, we changed the lower bound of the voltage range of the oscillating PD neurons by injecting an artificial dynamic-clamp outward current that activated when the membrane potential was below a predetermined threshold (also set to $\left.V_{\text {low }}\right)$. In this case, when the artificial current was activated, the lower bound of the PD oscillation was more hyperpolarized, whereas the upper bound remained unchanged (Fig. $3 B 1$ ). Different values of the maximal conductance of the dynamic clamp current resulted in different lower bound values. Again, as expected, when the lower bound of the oscillation was shifted to more negative values, $f_{\text {pyloric }}$ also decreased $(N=6 ; r=$ $0.61 ; p<0.01$ ) (Fig. 3B2). Overall, these results showed that changing the upper or lower bounds of the voltage range of the oscillating PD neuron shifted the $f_{\text {pyloric }}$ in the same direction that it shifted $f_{\max }$ in the previous set of experiments, consistent with our above-stated hypothesis.

The decision to set the threshold of the dynamic clamp current to $V_{\text {low }}$ was arbitrary. To see whether the value of the threshold voltage affected our results, we also examined the effect of dynamic-clamp currents with a different threshold value that was $10 \mathrm{mV}$ higher than the $V_{\text {low }}$ (the high threshold, $V_{\text {high }}$ ). Examples of low- versus high-threshold dynamic-clamp inward-current injections are shown in Figure $4, A$ and $B$. The inward dynamicclamp current with threshold at $V_{\text {high }}$ also effectively shifted the upper bound of the PD neuron oscillation to higher values without changing the lower bound. Interestingly, unlike the current injection with threshold at $V_{\text {low }}$, the high-threshold current injection did not change $f_{\text {pyloric }}$ despite increasing the upper bound $(N=6 ; r=0.06 ; p=0.67)$ (Fig. $4 C)$.

We also examined the effect of the threshold voltage in adjusting the lower bound of the PD oscillation with an outward artificial current. Once again, dynamic-clamp current injection could effectively decrease the lower bound of the PD oscillation without changing its upper bound, independent of the voltage threshold. However, in this case, the high-threshold dynamicclamp current acted similarly to the low-threshold current and decreased $f_{\text {pyloric }}(N=6 ; r=0.35073 ; p=0.01817)$.
A Low threshold

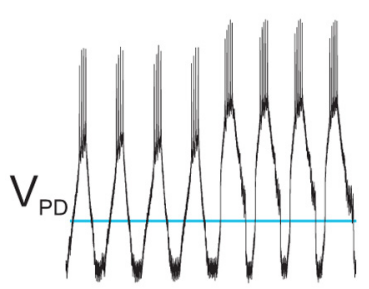

$10 \mathrm{mV}$

$10 \mathrm{nA}$

$1 \mathrm{~s}$
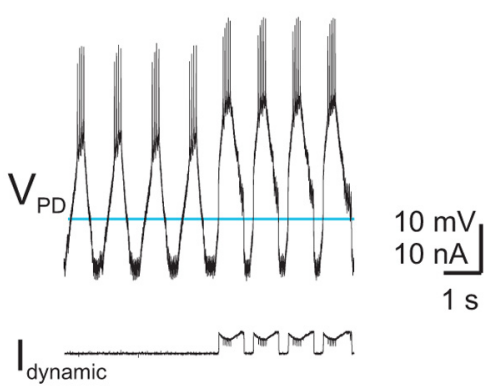

B High threshold

C

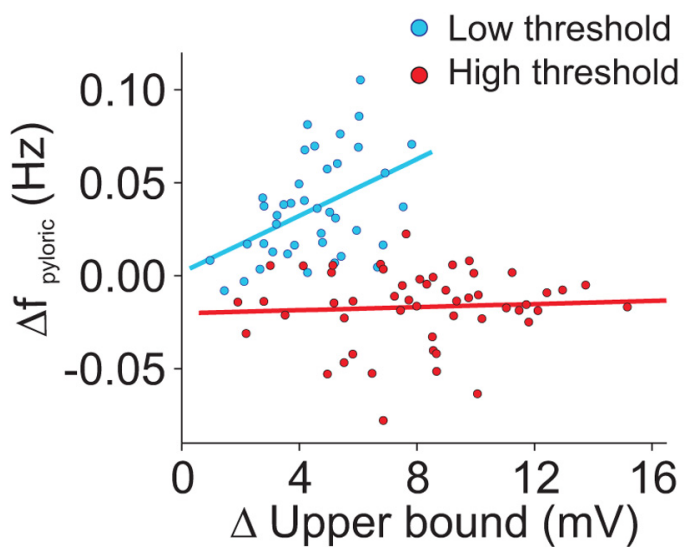

Figure 4. The effect of shifting the upper bound of the voltage oscillation is dependent on the activation threshold of the dynamic-clamp current. $\boldsymbol{A}, \boldsymbol{B}$, The upper bounds of the PD neuron oscillation are altered with low-threshold $(\boldsymbol{A})$ or high-threshold $(\boldsymbol{B})$ dynamic-clamp current. $\boldsymbol{C}$ Only the low-threshold dynamic-clamp current increased $f_{\text {pyloric, }}$ whereas the high-threshold dynamic-clamp current had no effect. The lines in $\boldsymbol{A}$ and $\boldsymbol{B}$ indicate the threshold voltage. The lines in $\boldsymbol{C}$ are the linear fits to the data points (low-threshold, $r=0.45, p<0.05$; highthreshold, $r=0.06, p=0.67$ ). Data points in ( are multiple runs in six experiments performed with different $g_{\max }$ values in dynamic clamp.

The difference between the effects of the dynamic-clamp inward current with low or high thresholds in the upper-bound shift experiments indicated that the PD neuron voltage range was not the only factor that affected $f_{\text {pyloric }}$. Because the dynamicclamp current, in addition to the voltage range, also changes the voltage waveform of the PD neuron, it is possible that some other properties of the waveform affected $f_{\text {pyloric }}$ in a way that could potentially oppose the shift due to increasing the upper bound. If so, according to our above-stated hypothesis, changes in the PD neuron waveform (that do not affect its voltage range) may also result in shifts of its preferred frequency $f_{\max }$.

\section{The effect of the PD neuron waveform on $f_{\max }$}

To explore the effect of the PD neuron waveform properties on $f_{\max }$ required waveforms with distinct properties. One possibility was to use artificial waveforms with distinct properties (e.g., different duty cycles, rise and fall slopes, etc.). Instead, we took advantage of the fact that the PD neurons in different preparations produce different waveforms during their natural oscillation. We selected seven PD neuron oscillation waveforms from different preparations and generated a group of representative realistic unitary waveforms from these recordings. To produce the unitary waveforms, each selected PD recording was filtered at $10 \mathrm{~Hz}$ to remove the action potentials (Fig. 5A) and the representative realistic waveforms were extracted from each of these filtered recordings (see Materials and Methods) (Fig. 5B, left). 
A
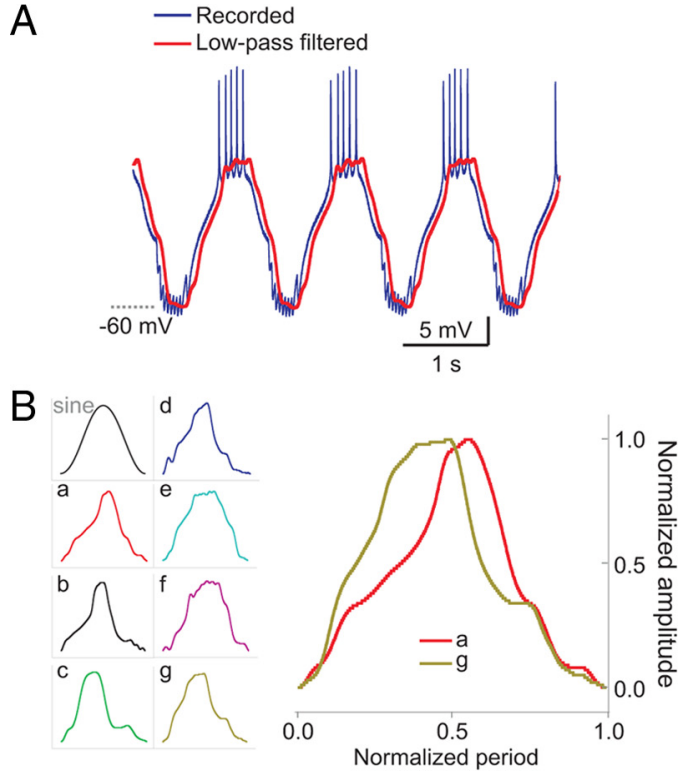

C
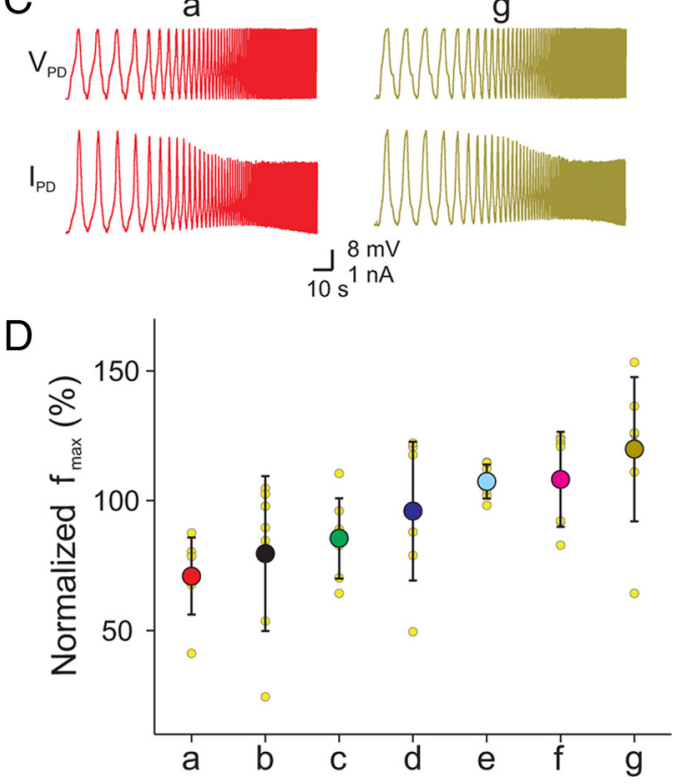

Figure 5. The preferred frequency depends on the oscillation waveform of the PD neuron. $A$, Selected PD recordings were filtered at $10 \mathrm{~Hz}$ to remove the action potentials and the resulting slow oscillation (red; shifted for clarity) was used to extract representative unitary waveforms. $\boldsymbol{B}$, Seven representative realistic unitary waveforms $(\boldsymbol{a}-\boldsymbol{g})$ were selected and compared with a sine wave (sine) in each of the seven PD neurons. The right panel shows an overlapped comparison of waveforms $\boldsymbol{a}$ and $\boldsymbol{g}$. $\boldsymbol{C}$, The recording of the membrane potential and injected current when the PD neuron was voltage clamped using a ZAP function built from the unitary waveform $\boldsymbol{a}$ or $\boldsymbol{g}$. $\boldsymbol{D}$, The preferred frequencies were measured with the representative realistic waveforms and normalized to the preferred frequency measured with the standard sinusoidal ZAP function. The waveforms are labeled and colored as in $\boldsymbol{B}$ and ordered in increasing averaged values of preferred frequency. Each of the yellow dots in $\boldsymbol{D}$ shows the measurement with that waveform type in a single experiment.

These waveforms differed in their duty cycle and slopes of rise and fall as well as other parameters (Table 1; Fig. $5 B$, right).

Each of the seven waveforms was used as a unitary waveform for generating a ZAP function (see Materials and Methods) and, in subsequent experiments, each PD neuron was voltage clamped with all these ZAP functions $(0.1-4 \mathrm{~Hz})$ as well as the standard sinusoidal ZAP. Two examples of the traces recorded from the PD neuron voltage clamped with the realistic waveform ZAP
Table 1. Parameters of the representative realistic waveform

\begin{tabular}{|c|c|c|c|c|c|c|c|c|}
\hline & \multicolumn{8}{|c|}{ Waveform } \\
\hline & $a$ & $b$ & C & $d$ & e & $f$ & $\mathrm{~g}$ & Sine \\
\hline Duty cycle & 0.495 & 0.439 & 0.36 & 0.508 & 0.6 & 0.508 & 0.454 & 0.500 \\
\hline Peak phase & 0.553 & 0.504 & 0.39 & 0.516 & 0.601 & 0.516 & 0.489 & 0.500 \\
\hline Area & 0.420 & 0.366 & 0.398 & 0.507 & 0.556 & 0.507 & 0.461 & 0.500 \\
\hline Portion $>75 \%$ & 0.193 & 0.172 & 0.247 & 0.386 & 0.374 & 0.386 & 0.27 & 0.333 \\
\hline Portion $<25 \%$ & 0.34 & 0.488 & 0.565 & 0.353 & 0.276 & 0.353 & 0.32 & 0.333 \\
\hline $0-100 \%$ slope & 1.81 & 1.99 & 2.57 & 1.94 & 1.67 & 1.94 & 2.05 & 2.00 \\
\hline $0-25 \%$ slope & 1.82 & 1.68 & 1.68 & 1.53 & 1.89 & 1.53 & 2.31 & 1.48 \\
\hline $25-50 \%$ slope & 1.27 & 1.31 & 4.72 & 2.21 & 3.73 & 2.21 & 3.01 & 2.98 \\
\hline $50-75 \%$ slope & 2.23 & 5.56 & 6.25 & 7.58 & 1.88 & 7.58 & 2.87 & 3.01 \\
\hline $75-100 \%$ slope & 2.36 & 2.12 & 1.70 & 1.21 & 0.93 & 1.21 & 1.19 & 1.52 \\
\hline $100-0 \%$ slope & -2.23 & -2.01 & -1.63 & -2.06 & -2.49 & -2.06 & -1.95 & -2.00 \\
\hline $100-75 \%$ slope & -2.84 & -4.55 & -2.48 & -1.38 & -2.34 & -1.38 & -4.10 & -1.52 \\
\hline 75-50\% slope & -5.21 & -5.43 & -6.41 & -3.25 & -2.84 & -3.25 & -5.10 & -3.01 \\
\hline $50-25 \%$ slope & -2.27 & -4.31 & -4.46 & -6.58 & -4.03 & -6.58 & -1.31 & -2.98 \\
\hline $25-0 \%$ slope & -1.24 & -0.74 & -0.60 & -0.97 & -1.75 & -1.32 & -1.18 & -1.48 \\
\hline
\end{tabular}

See Fig. $5 B$ for waveforms a- $g$.

function are shown in Figure $5 C$. We calculated the $f_{\max }$ as described above for all seven representative realistic waveforms and the standard sinusoidal ZAP. In these experiments, we were mainly interested in the effect of the waveform shape on $F$. A statistical analysis of all values measured indicated that this increase was highly significant $(N=7$; one-way ANOVA, $p<0.001)$.

What features of the PD neuron waveform are correlated with $f_{\max }$ ?

The representative realistic waveforms have different properties, including their duty cycle, the peak phase, the portions above or below certain threshold, and the slopes in different sections of the waveform. We were interested in which, if any, of these waveform characteristics correlated with the value of $f_{\max }$. For each waveform, we measured values of several characteristic parameters (see Materials and Methods) (Table 1). Surprisingly, most of these parameters showed only a weak correlation with the normalized $f_{\max }$ (Fig. 6; Table 2). For example, the duty cycle $(r=$ $0.18, p=0.17$ ) (Fig. $6 A)$ and the peak phase $(r=0.08, p=0.58)$ (Fig. $6 B$ ) had almost no correlation with $f_{\max }$. However, a few specific properties did stand out. In particular, the rising slope at $75-100 \%$ of the waveform amplitude showed a strong negative correlation $(r=-0.58)$ (Fig. $6 J)$ with the most statistically significant $p$ value $(<0.0001)$. In addition to the $75-100 \%$ rising slope, the total waveform area and the portion $>75 \%$ amplitude of the waveform also showed good correlation with $f_{\max }$ (area, $r=$ 0.43, $p<0.05$; portion $>75 \%, r=0.45, p<0.05$ ) (Fig. $6 C, D$ ). Altogether, the analysis of the waveform shape provided additional information on factors other than the upper and lower voltage range that affected the $f_{\max }$ value. In particular, when the top portion of the PD oscillation waveform was steeper, the PD neuron will had a smaller $f_{\max }$ value.

The PD neuron waveform shape in the ongoing pyloric oscillation is affected by dynamic-clamp currents

To examine whether any of the waveform parameters that showed a good correlation with $f_{\max }$ were predictive of the changes seen in $f_{\text {pyloric }}$ during the dynamic-clamp experiments, we measured how these parameters were affected by the dynamic-clamp currents. To analyze how the properties of the waveforms were changed by the low- or high-threshold dynamicclamp current injection in the upper-bound shift experiments, we compared the PD neuron waveforms during the current in- 
jection with those before the current injection in the same experiment. This comparison required us to generate representative waveforms for each recording, as described above. Examples of how the dynamic-clamp current injection altered the slow-oscillation waveform are shown in Figure 7, A (low-threshold) and $B$ (high-threshold).

We next explored how the shifts in the lower or upper bound of the voltage range during the dynamic-clamp experiments affected the three waveform parameters that showed a good correlation with $f_{\text {max }}$ : the $75-100 \%$ rising slope, the total waveform area, and the portion of the waveform $>75 \%$ amplitude. The effect of the dynamic-clamp currents was measured as the ratio of these values during the current injection to the values before injection in the same experiment and plotted against the shift in the upper bound of the voltage range. A ratio higher or lower than 1 means that the characteristic parameter increased or decreased, respectively, during current injection.

With the low-threshold dynamic-clamp current, the 75-100\% rising slope showed a slight decrease as the upper bound of the voltage range increased $(r=-0.36, p<$ 0.05) (Fig. 7C). In contrast, with highthreshold injection, the slope increased as the upper bound was increased $(r=0.39$, $p<0.05$ ) (Fig. 7C). The other two waveform parameters examined, total area and portion $>75 \%$ amplitude, increased with both low- and high-threshold dynamicclamp current injections, but the values for the low-threshold current were higher (Fig. $7 D, E$ ). The difference in the effects of the waveform parameters, especially the $75-100 \%$ rising slope, between the low- and high-threshold experiments could provide a possible explanation for the distinct effects on $f_{\text {pyloric }}$ in the upperbound shift experiments.

\section{Discussion}

A fundamental characteristic of oscillatory activity is its frequency. Different behavioral needs of the animal often require network oscillations to be active at different frequencies (Tryba et al., 2008); as such, it is important to know what factors determine this frequency. Modeling results show that many ionic currents can shape the preferred frequency of a neuron (Tohidi and Nadim, 2009) and even more currents potentially underlie oscillatory activity (Soto-Treviño et al., 2005). It is experimentally difficult, if not impossible, to measure every one of these ionic currents in the same neuron to determine their effects on the oscillation frequency. Measuring the membrane potential waveform, on the other hand, is relatively easy to do. The
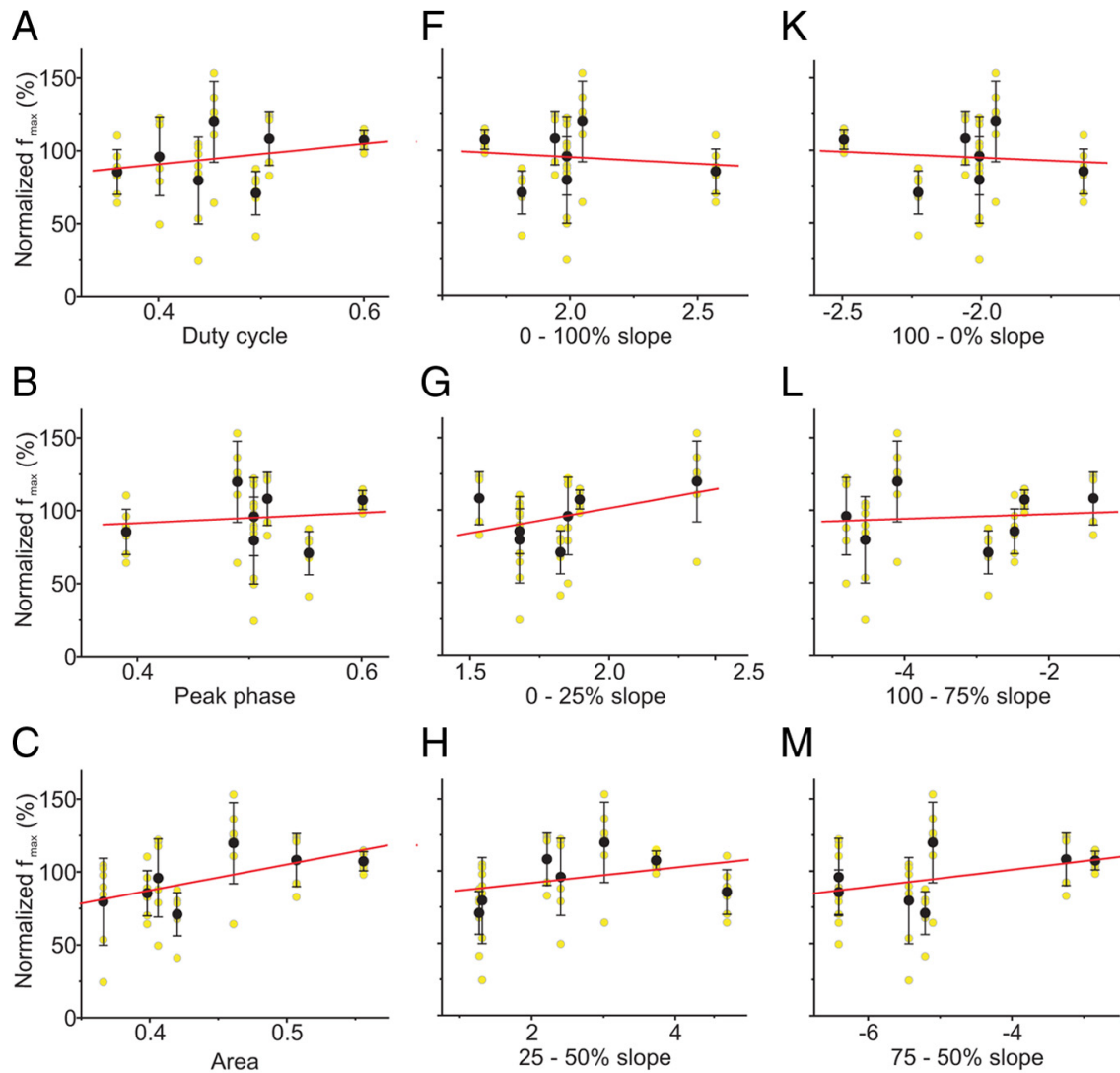

$\mathrm{H}$

$\mathrm{M}$
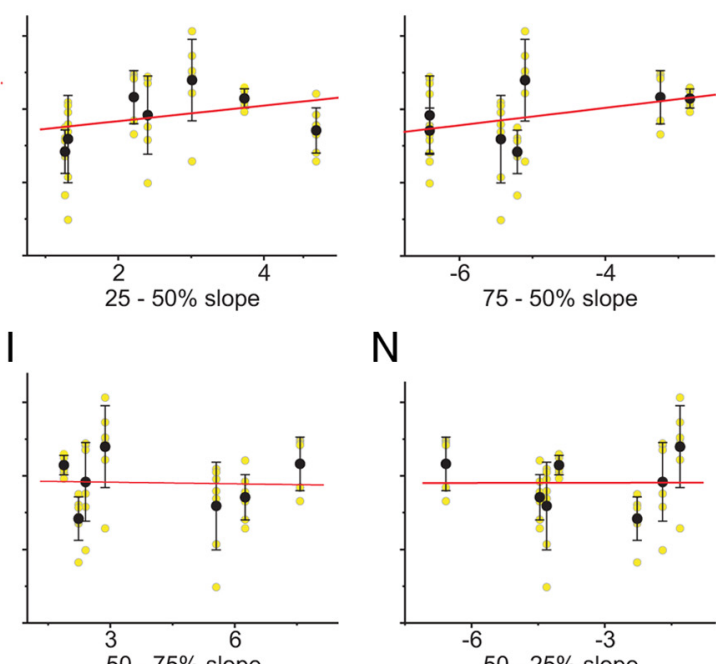

$\mathrm{N}$
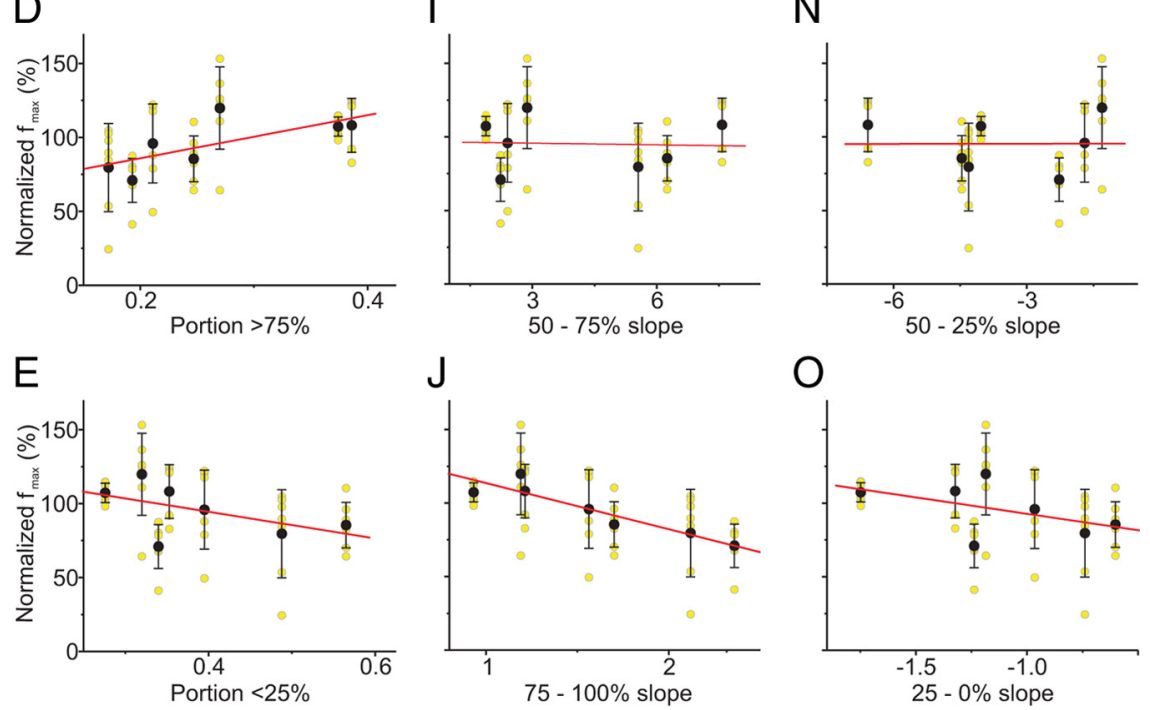

Figure 6. The correlations between the preferred frequency and various characteristics of the realistic waveforms. $\boldsymbol{A}-\mathbf{0}$, Fifteen properties of waveform were analyzed, including duty cycle $(\boldsymbol{A})$, peak phase $(\boldsymbol{B})$, area under the waveform $(\boldsymbol{C})$, portion $>75 \%(\boldsymbol{D})$, portion $<25 \%(\boldsymbol{E})$, and slope within different parts of the waveform $(\boldsymbol{F}-\mathbf{0})$. Among these properties, the rising slope at the 75-100\% amplitude $(\boldsymbol{J})$ showed the strongest (negative) correlation. Each yellow dot shows the measurement at that value of the parameter in a single experiment. Regression lines were fit to all data points, not to the mean values. Error bars show SD values. For detailed information see Table 2. 
Table 2. The correlations between different properties and the preferred frequency (linear fits, $y=a+b x$ )

\begin{tabular}{lccrr}
\hline & \multicolumn{1}{c}{$R$} & \multicolumn{1}{c}{$a$} & \multicolumn{1}{c}{$b$} \\
\hline Duty cycle & 0.19774 & 0.17322 & 62.84 & 69.82 \\
Peak phase & 0.08188 & 0.57597 & 77.50 & 35.08 \\
Area & 0.43171 & 0.00195 & 16.08 & 178.14 \\
$>75 \%$ & 0.44825 & 0.00124 & 56.79 & 145.57 \\
$<25 \%$ & -0.33165 & 0.01991 & 130.64 & -90.33 \\
$0-100 \%$ slope & -0.09362 & 0.52227 & 113.71 & -9.18 \\
$0-25 \%$ slope & 0.31046 & 0.02992 & 32.45 & 34.45 \\
$25-50 \%$ slope & 0.24113 & 0.0951 & 81.21 & 5.29 \\
$50-75 \%$ slope & -0.03032 & 0.83618 & 96.83 & -0.36 \\
$75-100 \%$ slope & -0.58792 & $<0.0001$ & 144.97 & -31.37 \\
$100-0 \%$ slope & -0.0771 & 0.59848 & 78.65 & -8.11 \\
$100-75 \%$ slope & 0.07492 & 0.60893 & 100.51 & 1.61 \\
$75-50 \%$ slope & 0.30256 & 0.0346 & 124.73 & 5.93 \\
$50-25 \%$ slope & 0.00362 & 0.98031 & 95.51 & 0.05 \\
$25-0 \%$ slope & -0.31024 & 0.03005 & 70.47 & -22.30 \\
\hline
\end{tabular}

We explored the parameters of the slow-oscillation waveform underlying the bursting activity of PD neurons, members of the crab pyloric pacemaker group, that affect their preferred frequency $\left(f_{\max }\right)$ and examined whether these parameters also change the network frequency $\left(f_{\text {pyloric }}\right)$. We found correlations between $f_{\max }$ and certain parameters that determine the waveform shape, including the lower and upper bounds of the voltage range and the peak rising slope. Shifting the voltage bounds with dynamic clamp during an ongoing oscillation produced shifts in $f_{\text {pyloric }}$ similar to those predicted by changes in $f_{\max }$. The dependence of $f_{\max }$ on the PD neuron waveform indicates that changes in the network frequency are reflected in corresponding changes in the waveform voltage range and other parameters.

\section{The effect of voltage range on preferred frequency}

Traditionally, preferred frequency is measured by injecting a ZAP-function current into a neuron to record its membrane potential, which results in membrane potential oscillations that vary in voltage range and amplitude (Puil et al., 1986; Hutcheon and Yarom, 2000). We measured the preferred frequency of the PD neuron, with both current- and voltage-clamp methods, using a range of membrane potential oscillations $(-60$ to $-30 \mathrm{mV})$ similar to the slow-wave of the bursting activity, and found similar values of $f_{\max }$ with both methods (Fig. 1). The voltage-clamp measurements demonstrated that $f_{\max }$ increases when the PD neuron oscillates with a higher voltage range, consistent with the effect of a DC-bias current (Tohidi and Nadim, 2009). In other systems, depolarizing the membrane potential may shift the $f_{\max }$ to higher (Gutfreund et al., 1995) or lower values (Hutcheon et al., 1996), but it is the presence of such a change that is important, not its direction.

The traditional description of membrane resonance involves the combination of the passive resistive and capacitive properties of the membrane together with at least one voltage-dependent current acting as an inductor (Hutcheon and Yarom, 2000). This standard resistor-inductor-capacitor circuit description of membrane resonance describes a linear phenomenon, whereby the voltage response scales with the amplitude of the input current and, therefore, the resonance frequency does not shift. Our results do not support this linear model. The significantly higher sensitivity of $f_{\max }$ to the shifts in the upper bound compared with the lower bound provides support for the nonlinearity of this phenomenon. Different ionic currents (e.g., $I_{\mathrm{Ca}}$ and $I_{\mathrm{h}}$ ) are often activated in the upper and lower envelopes of the voltage oscilla-
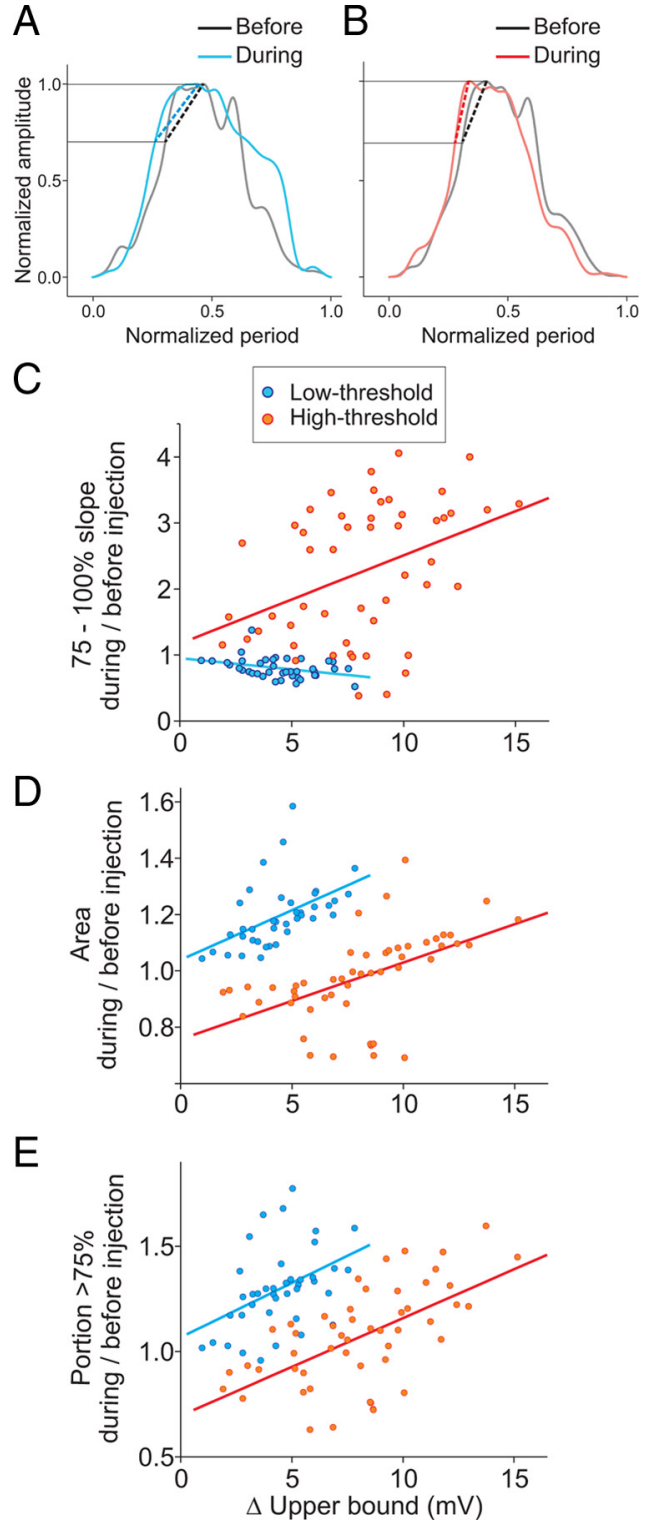

Figure 7. Parameters describing the PD neuron slow waveform depend on the dynamic-clamp current injection. $A, P D$ neuron waveforms before the low-threshold dynamic-clamp inward current injection (black) and during the injection (blue). Notice that the 75-100\% slope becomes somewhat shallower during injection (dashed line). $\boldsymbol{B}$, The waveforms before the high-threshold dynamic-clamp inward current injection (black) and during the injection (red). The 75-100\% slope became steeper during injection (dashed line). C, As the upper bound of the waveform increases, the $75-100 \%$ slope also increased during the high-threshold dynamic current injection. In contrast, the low-threshold current injection had almost no effect on the slope. Lines indicate linear fits to the data points (lowthreshold, $y=-0.034 x+0.952 ; r=-0.36, p<0.05$; high-threshold, $y=0.134 x+1.171 ; r=$ $0.39, p<0.05)$. $D$, The total area of the waveform increased with both low- and high-threshold dynamic clamp current injection (low-threshold, $r=0.51, p<0.001$; high-threshold, $r=0.53, p<$ $0.0001)$. However, the low-threshold current produced a larger waveform area. $\boldsymbol{E}$, The waveform portion $>75 \%$-amplitude also increased with both low-and high-threshold dynamic-clamp current injection (low-threshold, $r=0.43, p<0.005$; high-threshold, $r=0.60, p<0.0001$ ). However, the low-threshold current produced larger values. Data points in $\boldsymbol{C}-\boldsymbol{E}$ are multiple runs in six experiments performed with different $g_{\max }$ values in dynamic clamp.

tion, which may affect the value of $f_{\max }$ differently (Tohidi and Nadim, 2009). These currents have different activation properties, which may result in different sensitivities to the same amount of membrane potential change.

Conversely, it is important to understand which ionic currents shape the upper and lower limits of the voltage range. In a 
PD model neuron, increasing the conductance of $I_{\mathrm{h}}$ moves the baseline of the membrane potential to a more depolarized level and increases $f_{\max }$ (Tohidi and Nadim, 2009). Consistent with these results, our experiments show that an increase (i.e., depolarization) in the lower bound of the voltage range results in a higher $f_{\max }$. Another factor affecting the membrane potential in pyloric neurons is neuromodulation. Some modulators, such as dopamine, hyperpolarize the PD neuron whereas others, such as octopamine and pilocarpine, depolarize it (Marder and Eisen, 1984; Harris-Warrick et al., 1998; Kloppenburg et al., 1999; Goaillard et al., 2004). Most neuromodulators have an effect on the pyloric network frequency, but the influence of neuromodulators on the preferred frequency of pyloric neurons remains to be explored. The inhibitory LP-to-PD synapse is the only chemical feedback synapse from pyloric follower neurons to the pacemakers (Manor et al., 1997). The strength of the LP-to-PD synapse can be changed by neuromodulators, resulting in smaller or larger IPSPs and therefore moving the lower bound of the oscillation voltage range to a different value (Ayali et al., 1998; Thirumalai et al., 2006). Based on our results, we predict that this inhibitory synapse also has a role in controlling the frequency. However, previous studies have shown that blocking or enhancing this synapse (with neuromodulators) can have little effect on the pyloric network frequency (Nadim et al., 1999; Mamiya and Nadim, 2004; Thirumalai et al., 2006). One explanation is that the IPSP generated by the LP neuron under these situations is not large enough to produce an observable shift when it is removed or enhanced during the oscillation, particularly because much of the LP-induced IPSPs in the PD neuron are transient (Fig. $1 \mathrm{~A}$ ) and the dependence of $f_{\max }$ on the lower bound of the voltage range is significantly less sensitive than the upper bound (see Results). Alternatively, it is possible that other changes in the waveform shape of the PD neuron counteract the effect of the shift in the lower bound, resulting in a limited change in both $f_{\text {max }}$ and $f_{\text {pyloric }}$.

\section{Waveform shape and the determination of the network frequency}

The relationship between the parameters of waveform and the dynamics of ionic currents is bidirectional. For example, overexpression of $I_{\mathrm{h}}$ in the PD neuron changes its bursting waveform by increasing the duty cycle and number of action potentials per burst (Zhang et al., 2003), whereas the A-current delays the postinhibitory rebound (Tierney and Harris-Warrick, 1992), resulting in different delay times before the burst in pyloric constrictor neurons (Hooper, 1998; Zhang et al., 2008). The shape of the waveform also affects the dynamics of the voltage-gated ionic and synaptic currents. In leech heart interneurons, for example, voltage waveforms with different rise slopes produce different amounts of $I_{\mathrm{Ca}}$ and $I_{\mathrm{h}}$ (Olsen and Calabrese, 1996). When the waveform is altered, the resulting changes in the dynamics of ionic currents in turn change $f_{\max }$. Similarly, distinct realistic voltage waveforms in the pyloric network can result in different synaptic outputs, presumably due to their effect on the kinetics of presynaptic $\mathrm{Ca}^{2+}$ currents (Mamiya et al., 2003; Rabbah and Nadim, 2007).

Because $f_{\max }$ results from the dynamics of ionic currents, it can be assumed that it also depends on parameters that affect the neuron's waveform shape. The seven realistic PD neuron waveforms, when applied in the same voltage range, each produced a somewhat different value of $f_{\max }$. We ordered these waveforms according to their effect on $f_{\max }$ and examined how different waveform parameters correlated with these $f_{\max }$ values. Our goal was not to find independent parameters but to focus on those that provided intuitive distinctions between the waveforms. Of the 15 parameters examined, several were correlated with $f_{\max }$, but one, the $75-100 \%$ rising slope, showed the highest significance value and largest (negative) correlation.

We therefore focused on two important waveform characteristics that affected $f_{\text {max }}$ : the voltage range of oscillation and the $75-100 \%$ slope. We asked whether a combination of these two parameters explains the effect of the dynamic-clamp current on $f_{\text {pyloric }}$. During ongoing oscillations, the dynamic-clamp outward current shifts the lower bound of oscillations and results in a lower value of $f_{\text {pyloric }}$, as predicted by the effect of the lower bound on $f_{\max }$ (Fig. $3 B$ ), independent of its threshold. The dynamicclamp inward current also affected $f_{\text {pyloric }}$ by shifting the upper bound of oscillations, but this effect was only present with a lowbut not high-threshold current. Similar shifts in the upper bound of oscillations may occur in the biological setting as actions of low- or high-threshold activated inward currents, both of which have been shown to be activated in pyloric neurons by neuromodulators. For example, the modulator-activated inward current $I_{\mathrm{MI}}$, which is activated by a variety of peptides, has a low activation threshold (Golowasch and Marder, 1992; Zhao et al., 2010), whereas the calcium currents, which are subject to modulation by dopamine, have a much higher activation threshold (Johnson et al., 2003).

An examination of the PD waveform shape showed that the high- (but not low-) threshold current significantly increased the 75-100\% slope of the waveform. Whereas the increase in the upper bound will push $f_{\max }$ to a higher value, the increase in the $75-100 \%$ slope will decrease it. The combined effect of these two factors could produce little or no shift in the preferred frequency and, as a result, the network frequency, which is correlated with $f_{\max }$, would not change. Two other waveform parameters, total area and the portion $>75 \%$, that correlated positively with $f_{\max }$ increased with changes in the upper bound in both the low- and high-threshold current experiments. However, the values for the low-threshold experiments were higher, predicting a higher $f_{\max }$, which was consistent with the higher value of $f_{\text {pyloric }}$ measured in these experiments.

\section{Conclusions}

In other oscillatory systems, the network frequency is also similar to the preferred frequency of the neuron within the network (Leung and Yu, 1998; D'Angelo et al., 2001), although few studies have shown a direct correlation (Erchova et al., 2004; Schreiber et al., 2004). If the preferred frequency can be predictive of ongoing oscillations, it may be possible to extend our methodology to understand how parameters that shape the preferred frequency affect the network oscillation in many other systems. It should be noted, however, that the extent to which the parameters of one type of oscillatory neuron shape the network frequency may become more or less influential as a result of parallel changes in other neuron types in the network.

\section{References}

Ayali A, Johnson BR, Harris-Warrick RM (1998) Dopamine modulates graded and spike-evoked synaptic inhibition independently at single synapses in pyloric network of lobster. J Neurophysiol 79:2063-2069.

D’Angelo E, Nieus T, Maffei A, Armano S, Rossi P, Taglietti V, Fontana A, Naldi G (2001) Theta-frequency bursting and resonance in cerebellar granule cells: experimental evidence and modeling of a slow $k+-$ dependent mechanism. J Neurosci 21:759-770.

Erchova I, Kreck G, Heinemann U, Herz AV (2004) Dynamics of rat entorhinal cortex layer II and III cells: characteristics of membrane potential resonance at rest predict oscillation properties near threshold. J Physiol 560:89-110. 
Goaillard JM, Schulz DJ, Kilman VL, Marder E (2004) Octopamine modulates the axons of modulatory projection neurons. J Neurosci 24: 7063-7073.

Golowasch J, Marder E (1992) Proctolin activates an inward current whose voltage dependence is modified by extracellular $\mathrm{Ca}^{2+}$. J Neurosci 12: $810-817$.

Gutfreund Y, Yarom Y, Segev I (1995) Subthreshold oscillations and resonant frequency in guinea-pig cortical neurons: physiology and modelling. J Physiol 483:621-640.

Harris-Warrick RM, Johnson BR, Peck JH, Kloppenburg P, Ayali A, Skarbinski J (1998) Distributed effects of dopamine modulation in the crustacean pyloric network. Ann N Y Acad Sci 860:155-167.

Hooper SL (1998) Transduction of temporal patterns by single neurons. Nat Neurosci 1:720-726.

Hutcheon B, Yarom Y (2000) Resonance, oscillation and the intrinsic frequency preferences of neurons. Trends Neurosci 23:216-222.

Hutcheon B, Miura RM, Puil E (1996) Subthreshold membrane resonance in neocortical neurons. J Neurophysiol 76:683-697.

Johnson BR, Kloppenburg P, Harris-Warrick RM (2003) Dopamine modulation of calcium currents in pyloric neurons of the lobster stomatogastric ganglion. J Neurophysiol 90:631-643.

Kloppenburg P, Levini RM, Harris-Warrick RM (1999) Dopamine modulates two potassium currents and inhibits the intrinsic firing properties of an identified motor neuron in a central pattern generator network. J Neurophysiol 81:29-38.

Leung LS, Yu HW (1998) Theta-frequency resonance in hippocampal CA1 neurons in vitro demonstrated by sinusoidal current injection. J Neurophysiol 79:1592-1596.

Luther JA, Robie AA, Yarotsky J, Reina C, Marder E, Golowasch J (2003) Episodic bouts of activity accompany recovery of rhythmic output by a neuromodulator- and activity-deprived adult neural network. J Neurophysiol 90:2720-2730.

MacLean JN, Zhang Y, Johnson BR, Harris-Warrick RM (2003) Activityindependent homeostasis in rhythmically active neurons. Neuron 37:109-120.

Mamiya A, Nadim F (2004) Dynamic interaction of oscillatory neurons coupled with reciprocally inhibitory synapses acts to stabilize the rhythm period. J Neurosci 24:5140-5150.

Mamiya A, Manor Y, Nadim F (2003) Short-term dynamics of a mixed chemical and electrical synapse in a rhythmic network. J Neurosci 23:9557-9564.

Manor Y, Nadim F, Abbott LF, Marder E (1997) Temporal dynamics of graded synaptic transmission in the lobster stomatogastric ganglion. J Neurosci 17:5610-5621.

Marder E, Bucher D (2001) Central pattern generators and the control of rhythmic movements. Curr Biol 11:R986-R996.

Marder E, Calabrese RL (1996) Principles of rhythmic motor pattern generation. Physiol Rev 76:687-717.

Marder E, Eisen JS (1984) Electrically coupled pacemaker neurons respond differently to same physiological inputs and neurotransmitters. J Neurophysiol 51:1362-1374.
Nadim F, Manor Y, Kopell N, Marder E (1999) Synaptic depression creates a switch that controls the frequency of an oscillatory circuit. Proc Natl Acad Sci U S A 96:8206-8211.

Olsen OH, Calabrese RL (1996) Activation of intrinsic and synaptic currents in leech heart interneurons by realistic waveforms. J Neurosci 16:4958-4970.

Peck JH, Nakanishi ST, Yaple R, Harris-Warrick RM (2001) Amine modulation of the transient potassium current in identified cells of the lobster stomatogastric ganglion. J Neurophysiol 86:2957-2965.

Puil E, Gimbarzevsky B, Miura RM (1986) Quantification of membrane properties of trigeminal root ganglion neurons in guinea pigs. J Neurophysiol 55:995-1016.

Rabbah P, Nadim F (2007) Distinct synaptic dynamics of heterogeneous pacemaker neurons in an oscillatory network. J Neurophysiol 97:22392253.

Raper JA (1979) Nonimpulse-mediated synaptic transmission during the generation of a cyclic motor program. Science 205:304-306.

Schreiber S, Erchova I, Heinemann U, Herz AV (2004) Subthreshold resonance explains the frequency-dependent integration of periodic as well as random stimuli in the entorhinal cortex. J Neurophysiol 92:408-415.

Soto-Treviño C, Rabbah P, Marder E, Nadim F (2005) Computational model of electrically coupled, intrinsically distinct pacemaker neurons. J Neurophysiol 94:590-604.

Thirumalai V, Marder E (2002) Colocalized neuropeptides activate a central pattern generator by acting on different circuit targets. J Neurosci 22:1874-1882.

Thirumalai V, Prinz AA, Johnson CD, Marder E (2006) Red pigment concentrating hormone strongly enhances the strength of the feedback to the pyloric rhythm oscillator but has little effect on pyloric rhythm period. J Neurophysiol 95:1762-1770.

Tierney AJ, Harris-Warrick RM (1992) Physiological role of the transient potassium current in the pyloric circuit of the lobster stomatogastric ganglion. J Neurophysiol 67:599-609.

Tohidi V, Nadim F (2009) Membrane resonance in bursting pacemaker neurons of an oscillatory network is correlated with network frequency. J Neurosci 29:6427-6435.

Tryba AK, Peña F, Lieske SP, Viemari JC, Thoby-Brisson M, Ramirez JM (2008) Differential modulation of neural network and pacemaker activity underlying eupnea and sigh-breathing activities. J Neurophysiol 99:2114-2125.

Zhang Y, Oliva R, Gisselmann G, Hatt H, Guckenheimer J, Harris-Warrick RM (2003) Overexpression of a hyperpolarization-activated cation current (Ih) channel gene modifies the firing activity of identified motor neurons in a small neural network. J Neurosci 23:9059-9067.

Zhang Y, Bose A, Nadim F (2008) Predicting the activity phase of a follower neuron with A-current in an inhibitory network. Biol Cybern 99:171-184.

Zhao S, Golowasch J, Nadim F (2010) Pacemaker neuron and network oscillations depend on a neuromodulator-regulated linear current. Front Behav Neurosci 4:21. 